\title{
A REVIEW ON FORMULATION AND CHARACTERIZATION OF HERBOSOME COMPLEX
}

\author{
IMDADUL HAQUE CHAUDHURY ${ }^{*}$, PULAK DEB ${ }^{2}$, 3 SUMIT DAS
}

1,2Department of Pharmaceutics, Girijananda Chowdhury Institute of Pharmaceutical Science, Hatkhowapara, Azara, Guwahati-17, ${ }^{3}$ Department of Pharmaceutical Chemistry, Girijananda Chowdhury Institute of Pharmaceutical Science, Azara, Hatkhowapara, Guwahati-17

Email: imdadbeing007@gmail.com

Received: 20 Apr 2019, Revised and Accepted: 26 Jun 2019

\begin{abstract}
The major amount of active constituents comprises in herbal drugs with excellent bioactivity in vitro but less in vivo because of their poor lipid solubility and improper size of the molecules. This results in poor absorption and bioavailability of active constituents from the herbal extract. Herbosome technology enhances the bioavailability of herbal extracts. Herbosome act as the bridge between the novel delivery system and conventional delivery system. It is a complex of natural active ingredients and phospholipids (phosphatidylcholine, phosphatidylserine etc.) which increases absorption of herbal extract. Herbosome is the novel emerging technique applied to phytopharmaceuticals for the enhancement of bioavailability of herbal extract for medicinal applications. This article overviews about herbosome technology, recent advance, their application for various standardized herbal extracts and aims to provide complete scientific information, characterization about herbosomes as a promising drug delivery system.
\end{abstract}

Keywords: Herbosomes, Phospholipid, Flavanoids, Phytomedicine

(C) 2019 The Authors. Published by Innovare Academic Sciences Pvt Ltd. This is an open access article under the CC BY license (http://creativecommons.org/licenses/by/4.0/)

DOI: http://dx.doi.org/10.22159/ijcpr.2019v11i4.34948

\section{INTRODUCTION}

Since ancient times the therapeutic uses of traditional medicines and phytomedicines have proved very popular for health maintenance by various means. The advancement in the field of herbal drug delivery started recently with the aim to manage human diseases efficiently [1]. Every nation is seeking health care beyond the traditional boundaries of modern medicine; turning to selfmedication in the form of herbal remedies. Most of the bioactive constituents of phytomedicines are water-soluble molecules (e. g. Phenolics, glycosides, flavonoids etc.). However, water-soluble phytoconstituents are limited in their effectiveness because they are poorly absorbed when taken orally or when applied topically. Many approaches have been developed to improve the oral bioavailability, such as the inclusion of solubility and bioavailability enhancer, structural modification and entrapment with the lipophilic carriers and thus extensive research in the field of herbal drug delivery systems as a means of improving the therapeutic indices of drugs is inevitable. The use of formulation technology to deliver herbal products and drugs by improved absorption and, as a consequence, produce better results than those obtained by conventional herbal extracts. Herbosome technology is a breakthrough model for marked enhancement of bioavailability, significantly greater clinical benefit, assured delivery to the tissues, without compromising nutrient safety [2].

The term "herbo" means plant, while "some" means cell-like. Most of the biologically active constituents of plants are polar or watersoluble molecules. However, water-soluble phytoconstituents (like flavonoids, tannins, glycoside aglycones, etc) are poorly absorbed either due to their large molecular size which cannot absorb by passive diffusion or due to their poor lipid solubility; severely limiting their ability to pass across the lipid-rich biological membranes, resulting in poor bioavailability [3]. Herbosomes are an advanced form of herbal product in combination with phospholipids having better absorption and utilization profile in our body and subsequently produce better therapeutic efficiency than conventional herbal extract or individual molecule, which can minimize the shortcoming of conventional therapy [4].

The drug formulations of traditional systems of medicine like the African, Chinese and Indian systems usually contain crude extracts of different herbs which incorporate in the undesirable and many times, toxic principles along with the active principles. With the developments in the field of phyto and analytical chemistry, specific ingredients or a group of similar ingredients from plants are being extracted, isolated and tested for their different therapeutic applications. Nevertheless, isolation and purification of individual components from whole herbal extracts often lead to partial or total loss of therapeutic activity. Although having excellent bioactivity in vitro, plant extracts often exhibit poor effectiveness in vivo or in animal models. The basic reasons for the low bioavailability of herbal extracts are that the bioactive components of these herbs possess multi-ring molecular structures which cannot be absorbed into the blood by simple passive diffusion and the bioactive phytoconstituents are mostly water soluble, hence, their poor lipid solubility limits their ability to pass across lipid biomembranes. Moreover, when it is taken orally bioactive phytoconstituents are destroyed by or lost to the gastric environment or they may be rendered less effective by interaction with other drugs or nutraceuticals [5, 6].

\section{Properties of herbosome}

Herbosomesare complex between a natural phytoconstituents and natural phospholipids, like soy phospholipids mostly phosphatidylcholine. These complex results from the reaction of stoichiometric amounts of phospholipids with the phytoconstituents in an aprotic solvent.

Herbosomescan accommodates the active principle that is anchored to the polar head of the phospholipids, which finally becomes an integral part of the membrane. Herbosomesare advanced form of herbal drugs which are better absorbed, utilized and which finally leads to better results than conventional dosage form. The increased bioavailability has been demonstrated by the pharmacokinetic studies as well as by pharmacokinetic tests in experimental animals and human subjects.

Herbosomesare lipophilic substances with the definite melting point, freely soluble in non-polar solvents, and moderately soluble in fats. Herbosomeswhen treated with water assume a micellar shape, forming the structure that resembles liposomes exhibiting fundamental difference [7]. 


\section{Methods of herbosome preparation}

Herbsomes novel complexes which are prepared by reacting from 32 moles but preferably with one mole of natural or synthetic phospholipids like phosphatidylcholine, phosphatidylethanolamine or phosphatidylserine with one mole of component like flavolignans, either alone or in the natural mixture in aprotic solvents such as dioxane or acetone. The herbosome complex can be then isolated by precipitation with non-solvent such as aliphatic hydrocarbons or lyophilization or by spray drying. In the complex formation of herbosomes, the ratio between these two moieties is in the range from 0.5-2.0 moles. The most preferable ratio of phospholipids to flavonoids is $1: 1[7,8]$.

\section{Anti-solvent precipitation technique:}

The specific amount of plant extract and phospholipid were taken into a $100 \mathrm{ml}$ round bottom flask and refluxed with $20 \mathrm{ml}$ of dichloromethane at a temperature not exceeding $60^{\circ} \mathrm{C}$ for $2 \mathrm{~h}$. The mixture is concentrated to $5-10 \mathrm{ml}$. Hexane $(20 \mathrm{ml})$ was added carefully with continuous stirring to get the precipitate which was filtered and collected and stored in desiccators overnight. The dried precipitate is crushed in a mortar and sieved through \#100 meshes. Powdered complex was placed in an amber colored glass bottle and stored at room temperature.

\section{Rotary evaporation technique}

The specific amount of plant material and phospholipid were dissolved in $30 \mathrm{ml}$ of tetrahydrofuran in a rotary round bottom flask followed by stirring for 3 hours* at a temperature not exceeding $40^{\circ} \mathrm{C}$. Thin film of the sample was obtained to which n-hexane was added and continuously stirred using a magnetic stirrer. The precipitate obtained was collected, placed in an amber colored glass bottle and stored at room temperature.

\section{Solvent evaporation technique}

The specific amount of plant material and phospholipids were taken into a $100 \mathrm{ml}$ round bottom flask and refluxed with $20 \mathrm{ml}$ of acetone at a temperature $50-60^{\circ} \mathrm{C}$ for $2 \mathrm{~h}$. The mixture is concentrated to 5 $10 \mathrm{ml}$ to obtain the precipitate which was filtered and collected. The dried precipitate phytosome complex was placed in an amber colored glass bottle and stored at room temperature.

\section{Ether-injection technique}

In this technique, the drug-lipid complex is dissolved in an organic solvent. This mixture is then slowly injected into a heated aqueous agent, resulting in the formation of vesicles. The state of amphiphiles depends on concentration. When the concentration is less, amphiphiles introduce a monomer state but as the concentration is increased, variety of structures may be formed, that is, round, cylindrical, disc, cubic, or hexagon type.

\section{Mechanism of working}

Phospholipids are amphipathic in nature they have both polar as well as non-Polar Regions. Their polar end consists of amine or phosphate groups which bound to the substrates polar group via weak hydrogen bonds and the remaining non-polar chain of the phospholipid warps itself over the formed complex thereby imparting a lipophilic character to the complex. Now it can easily pass through the lipophilic enterocyte membrane and the passage of the botanical derivative (in the form of a complex with phospholipid) through the GIT barrier become easy (water phase>enterocyte $>$ systemic circulation) $[9,10]$.

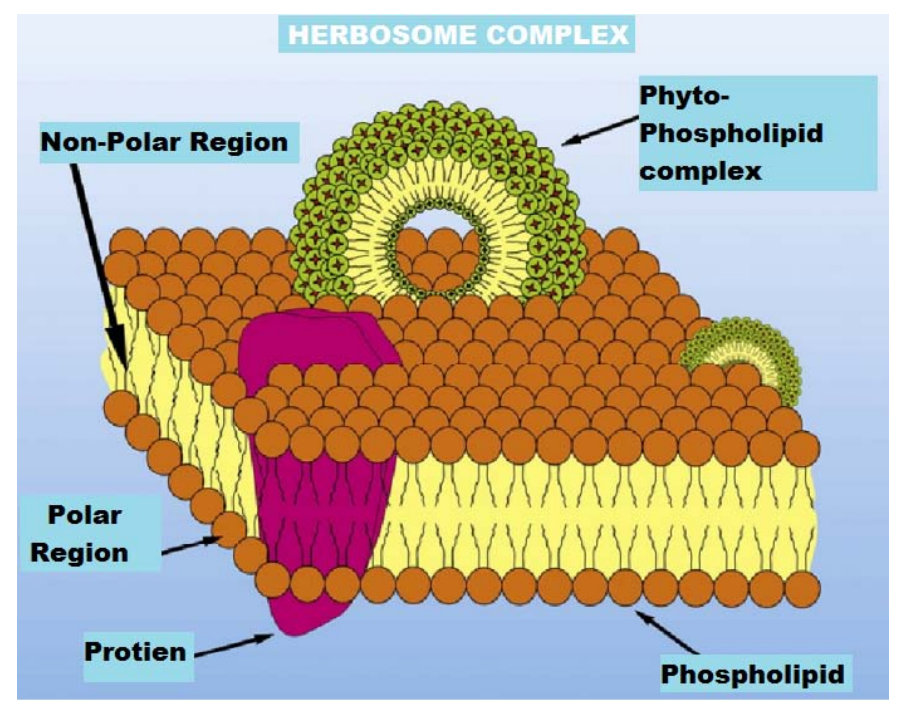

Fig. 1: Mechanism of herbosome loaded complex

\section{Herbosomes and liposomes: a comparision}

Liposomes are also prepared by mixing suitable water-soluble phytoconstituents in phosphatidylcholine in a definite ratio under suitable conditions. Here no chemical bond is formed, the phosphatidylcholine moiety just anchors the water-soluble phytoconstituents as a result of which there may be hundreds or even thousands of phosphatidylcholine molecules surrounding the drug molecule. In case of herbosomes the phosphatidylcholine and the plant constituents form a complex in the ratio $1: 1$ or $2: 1$ and the process of phytosome formation involves chemical bond formation whereas the liposomes are completely devoid of the chemical bond formation between the phosphatadylcholine molecule and the phytoconstituents. Due to the lesser composition of the phospholipid content in case of phytosomes the phytosomes are more bioavailable and are absorbed to a better extent than the liposomes [11].

\section{Merits and demerits of herbosomes}

\section{Merits of herbosomes}

Herbosomes show better stability as the chemical bond is formed between phospholipid molecule and phytoconstituent. Dose of phytoconstituents is reduced due to more bioavailability of phytoconstituents in the complex form. Duration of action is increased and also Herbosomes are simple to manufacture. Phytoconstituents complex with phospholipids are more stable in gastric secretion and resist the action of gut bacteria. Herbosome formulation technique enhanced the permeability of phytoconstituents across the biological membranes $[11,12]$. 


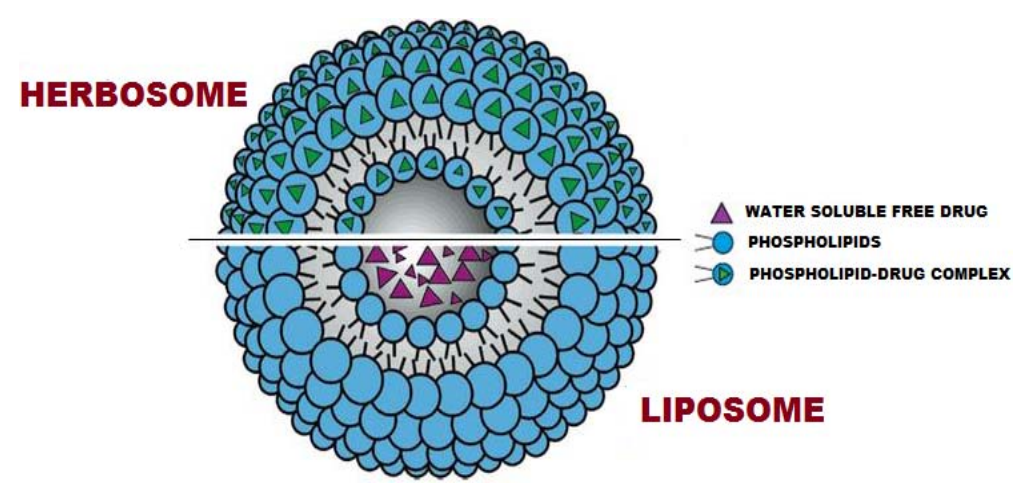

Fig. 2: Herbosomesvs liposomescomparision

\section{Demerits of herbosomes}

There are also some few demerits in an herbosome formulation as the phytoconstituents of herbosome are rapidly eliminated. Herbosomes shows short half-life. Hydrolysis, fusion, leakage and oxidation is undergone by the phospholipids. It has a high cost of production and sometimes the occurrence of allergic reactions to the herbosomal constituents may be observed. Because of their larger size problems can occur while trying to target to the various tissues.

\section{Application of herbosome}

Herbosomes are used in the treatment of various diseases like liver disease and heart disease. It is also used as an anti-inflammatory, lipolytic, vasokinetic, anti-oedema, cicatrizing, trophodermic, neutraceutical immunomo-dulator, antioxidant for skin and liver, cardioprotective, anti-wrinkles and UV protectant.

The fruit of milk thistle plant contains a flavonoid known for hepatoprotective effect. Silymarin has been shown to have a positive effect in treating liver diseases of various kinds, including hepatitis, cirrhosis, fatty filtration of liver and inflammation of bile duct. Silybin protects the liver by conserving glutathione into the Parenchyma Cells. While Parenchyma cell (PC) helps repair and replace the cell membrane. These constituents likely offer the synergistic benefit of sparing liver cell from destruction [13].

Studies have shown that gingko phytosomes (prepared from the standardized extract of Ginkgo Biloba leaves) produced better results compared to the conventional standardized extract from the plant (GBE, 24\% ginkgo flavones glycoside and 6\% terpenes lactones). In a bioavailability study conducted with healthy human volunteers, the level of GBE constituents (flavonoids and terpenes) from the Phytosomal form peaked after 3 hours* and persisted longer for at least 5 hours* after oral administration. It was found that the Phytosomal GBE produced a 2-4 times greater plasma concentration of terpenes than did the non-Phytosomal GBE. Its major indication is cerebral insufficiency and peripheral vascular disorders and it can also ameliorate reduced cerebral circulations. Its improved oral bioavailability and good tolerability makes it the ideal gingko product even for long term treatment. Studies have also proved the improve efficacy of ginkgo phytosomes over the conventional standardized extract in protecting rat isolated hearts against ischemia [14].

Most of the Phytosomal studies are focused on Silybummarianum (milk thistles) which contains premier liver protectant flavonoids. In 2006 Yanyu prepared silymarinphytosome and studied its pharmacokinetics in rats. In the studies, the bioavailability of silybin in the rat was increased remarkably after oral administration of silybin-phospholipid complex due to an impressive improvement of the lipophilic properties of silybin-phospholipid complex and improvement of the biological effect of silybin [15].

Grape seed phytosome composed of oligomeric polyphenols of varying molecular size, complexed with phospholipids. The main properties of procyanidin flavonoids of grape seed are, increase in total antioxidant capacity and stimulation of physiological antioxidant defenses of plasma, protection against ischemia/refusion induced damages in the heart, and protective effects against atherosclerosis thereby offering marked protection for the cardiovascular system and other organs through a network of mechanisms that extend beyond their great antioxidant potency [16].

Green tea has got several long term beneficial activities such as antioxidant, anticarcinogenic, antimutagenic, antiatherosclerotichypocholesterolemic, cardioprotective and antibacterial effect. Despite such potential action green tea polyphenols have very poor oral bioavailability from conventional extracts. The complexion of green tea polyphenols with phospholipids strongly improves their poor oral bioavailability [17].

\section{Characterization of herbosomes}

\section{Physical attributes}

The following are the characterization techniques used for Phytosomes in characterizing its physical attributes [18].

\section{Visualization}

Visualization of phytosomes can be achieved using transmission electron microscopy (TEM) provides the details about the internal composition and can show many characteristics of the phytosomes, such as morphology, crystallization, stress or even magnetic domains. Scanning electron microscopy (SEM) focuses on the phytosomes surface and its composition provides morphological details.

\section{Particle size and zeta potential}

The particle size and zeta potential can be determined by dynamic light scattering (DLS) using a computerized inspection system and photon correlation spectroscopy (PCS).

\section{Entrapment efficiency}

The entrapment efficiency, capability of the drug to be entrapped in phytosomes can be measured by the ultracentrifugation technique. It gives an idea about the $\%$ drug that is successfully entrapped into the phytosomes.

\section{Transition temperature}

The transition temperature of the vesicular lipid systems can be determined by differential scanning calorimetry.

\section{Surface tension activity measurement}

The surface tension activity of the drug in aqueous solution can be measured by the ring method in a Du Nouy ring tensiometer.

\section{Vesicle stability}

The stability of vesicles can be determined by assessing the size and structure of the vesicles over time. The mean size is measured by DLS and structural changes are monitored by TEM.

\section{Drug content}

The amount of drug can be quantified by a modified high performance liquid chromatographic method or by a suitable spectroscopic method. 


\section{Spectroscopic evaluation}

The spectroscopic evaluations are widely employed in order to confirm the formation of complex between phytoconstituents and the phospholipids moiety as well as to study the corresponding interaction between the two [18].

\section{${ }^{1}$ H-NMR}

The complex formation between the active phytoconstituents and the phosphatidylcholine molecule can be estimated by this method.

\section{${ }^{13} \mathrm{C}$-NMR}

In the ${ }^{13} \mathrm{C}$ NMR of the phytoconstituents and the stoichiometric complex with the phosphatidylcholine when recorded the phytoconstituents carbons were invisible. The signals corresponding to the glycerol and choline portion are broadened and some are shifted, while most of the resonance of the fatty acid chains retains their original sharp line shape.

\section{FTIR}

The formation of the complex can be confirmed by IR spectroscopy, comparing the spectrum of the complex with the spectrum of the individual components and their mechanical mixtures. FTIR spectroscopy is also a useful tool for the control of the stability of phytosomes when micro-dispersed in water or when incorporated in very simple cosmetic gels. From a practical point of view, the stability can be confirmed by comparing the spectrum of the complex in solid form (herbosomes) with the spectrum of its microdispersion in water after lyophilization, at different times.

\section{In vitro and in vivo evaluations}

Models of in vitro and in vivo evaluations are selected on the basis of the expected therapeutic activity of the biologically active phytoconstituents present in the herbosomes.

\section{Herbosome formulations}

Herbosomecomplexes can be converted and formulated in different kinds of dosage forms used both orally and topically. Various products can be designed in order to obtain the best performances of this technological innovation both in terms of formulating manageability and enhanced bioavailability.

\section{Soft gelatin capsules}

Soft gelatin capsules represent an ideal solution to formulate herbosomescomplexes. The herbosome complex can be dispersed in oily vehicles to obtain suspensions to be filled in soft gelatin capsules. Vegetable or semi-synthetic oils can be used to this purpose. Indena recommends a granulometry of $100 \%<200 \mu \mathrm{m}$ to best perform capsule production. According to Indena experience, not all the phytosome complexes behave in the same way when dispersed in oily vehicles and when the oily suspension is filled in the soft gelatin capsules; for this reasons preliminary feasibility trials should be performed to select the most suitable vehicle [19].

\section{Hard gelatin capsules}

The Phytosome complex can be formulated in hard gelatin capsules as well. A direct volumetric filling process (without precompression) can be applied, even if the apparently low density of the phytosome complex seems to limit the maximum amount of powder that can be filled into a capsule (usually not more than $300 \mathrm{mg}$ for a size 0 capsule). With a piston tamp capsule filling process, however, it is possible to increase the amount of powder which can be filled in a capsule, but precompression might affect the disintegration time. Indena recommend to careful monitoring the related parameters during product/process development. A preliminary dry granulation process is advisable, defines the best manufacturing process [20].

\section{Tablets}

Dry granulation represents the ideal manufacturing process to obtain tablets with higher unitary doses and with suitable technological and biopharmaceutical properties. However, due to the limited flowability, potential stickiness and low apparent density of the phytosome complex, a direct compression process can be applied only for low unitary doses; note that whenever a direct compression process is applied, the phytosome complex should be diluted with $60-70 \%$ of excipients to optimize its technological properties and to obtain tablets with appropriate technological and biopharmaceutical characteristics. On the other hand, wet granulation should be avoided due to the negative effect of water and heat (granulation/drying) on the stability of the phospholipid complex [21].

\section{Topical dosage forms}

The herbosome complex can be formulated topically as well. The ideal process to incorporate the herbosome complex in emulsion is to disperse the phospholipidic complex in a small amount of the lipidic phase and add it to the already created emulsion at low temperatures (not higher than $40^{\circ} \mathrm{C}$ ). The herbosomecomplexes are dispersible in the main lipidic solvents employed in topical formulations. In case of formulations containing a limited amount of lipids, the phytosome complex might also by dispersed into the watery phase, and again adde added to the final formulation at a temperature lower than $40{ }^{\circ} \mathrm{C}[22]$.

\section{CONCLUSION}

Herbosomes are an advanced form of herbal extract that are absorbedbetter than conventional herbal extract. The article thus reviews the benefits, physical characteristics, chemical properties, and method of preparation of herbosomes. The formulation methodology for phytosome is simple and can be easily upgraded to a commercial scale. These are novel complexes showing much better absorption profile following oral administration owing to improved lipid solubility which enable them to cross the biological membrane, resulting in enhanced bioavailability i.e. more amount of active principle in the systemic circulation. Also, phytosomes are superior to liposomes due to much better absorption and stability profile. As mentioned in the literature, phytosomes have been therapeutically used for hepatoprotective and liver diseases. After screening and selection of herbal extracts, one can develop Phytosomal drug delivery systems for various drug categories like anticancer, cardiovascular, and anti-inflammatory activities, etc.

\section{AUTHORS CONTRIBUTIONS}

All the author have contributed equally

\section{CONFLICT OF INTERESTS}

\section{Declare none}

\section{REFERENCES}

1. Gold J, Laxer D, Rochon P. Herbal remedies; a critical perspective. Ann R Coll Physician Surg Can 2000;33:497-8.

2. Kumari P, Singh N, Cheriyan P, Neelam. Phytosome: a noval approach for phytomedicine. Int J Institutional Pharm Life Sci 2011;1:89-100.

3. Kumar VS. Herbosome, novel carrier for herbal drug delivery. Curr Pharm Res 2007;3:24-27.

4. Patel J, Patel R, Khambholja K. An overview of phytosomes as an advanced herbal drug delivery system. Asian J Pharm Sci 2009;4:363-71.

5. Khan A, Alexander J, Ajazuddin A. Recent advances and future prospects of phytophospholipidcomplexation technique for improving the pharmacokinetic profile of plant actives. Int J Health Res 2013;168:50-60.

6. Bhattacharya S. Phytosomes: the new technology for enhancement of bioavailability of botanicals and nutraceuticals. Int J Health Res 2009;2:225-32.

7. Rudra PS, Ramakant N. Preparation and evaluation of phytosome of lawsone. Int J Pharm Sci Res 2015;12:5217-26.

8. Prachi U, Omprakash B. Phyto-phospholipid complex vesicles for phytoconstituents and herbal extracts: a promising drug delivery system. Int J Herbal Med 2016;4:14-20.

9. U Citernesi, M Sciacchitano. Phospholipids active ingredient complexes. Cosm Toil 1995;110:57-68.

10. Ravi GS, Chandur V, Shabaraya ARK. Phytosomes: an advanced herbal drug delivery system. IJPRBS 2015;4:415-32.

11. Bombardelli E, Mustich G. Bilobalide phospholipid complex, their uses and formulation containing them, U. S. Patent; 1991. 
12. Kidd PM. Phosphatidylcholine: a superior protectant against liver disease. Alternative Med Rev 1996;1:258-74.

13. Valenzuela A, Aspillaga M, Guerra R. Selectivity of silamarin on the increase of glutathione containing in different tissues of Rat. Plant Med 1989;55:42.

14. Maiti K, Kakali M, Arunava G, Bishnu PS, Pulok KM. Curcumine phospholipid complex: Preparation,therapeutic evaluation and pharmacokinetic study in rats. Int J Pharm 2007;330:155-63.

15. Singh RP, Parpani S, Narke R, Chavan R. Phytosome: recent advance research for novel drug delivery system. Asian J Pharm Res Dev 2014;2:15-29.

16. Tedesco D, Steidler S, Galletti S, Tameni M, Sonzogni O, Ravarotto L. Efficacy of silymarin-phospholipid complex in reducing the toxicity of aflatoxin B1 in broiler chicks. Poult Sci 2004;83:1839-43.

17. Hikino H, Kiso Y, Wagner H, Fiebig M. Antihepatotoxic actions of flavonolignans from silybummarianum fruits. Planta Med 1984;50:248-50.
18. Acharya NS, Parihar GV, Acharya SR. Phytosomes: a novel approach for delivering herbal extract with improved bioavailability. Int J Pharm Sci 2011;2:144-60.

19. Pallav Kaushik Deshpande, Anupam Kumar Pathak, Ragini Gothalwal. Phytosomes: a noval drug delivery system for phytoconstituents. J New Biol Reports 2014;3:212-20.

20. Nayyer Karimi, Babak Ghanbarzadeh, Hamed Hamishehkar, Akram Pezeshki, Hadi Mostafayi, Mohammad Mahdi Gholian. Phytosome as novel delivery system for nutraceutical materials. Int J Curr Microbiol Appl Sci 2015;4:152-9.

21. Sandeep Arora. Preparation and characterization of phytosomal-phospholipid complex of P. amarus and its tablet formulation. J Pharm Technol Res Management 2013;1:1-18.

22. Bhupen Kalita, Malay $\mathrm{K}$ Das. Resveratrol-phospholipid complexes (phytosomes) with improved physicochemical properties favorable for drug delivery via the skin. World J Pharm Res 2015;4:1497-517. 